\title{
Semantic prime inhibition and memory monitoring
}

\author{
ALAN S. BROWN and CARRIE K. BRADLEY \\ Southern Methodist University, Dallas, Texas
}

\begin{abstract}
The influence of semantically related primes (non-capital cities) on subsequent feeling of knowing (FOK), recall, and recognition of targets (capital cities) was examined in two studies. Subjects first rated their familiarity with 84 non-capital cities (primes). Each state had four, two or no cities presented in this task. After this, subjects rated their FOK for state capital recall, then recalled the state capitals, and finally selected the state capital from five different cities from that state. Both investigations revealed a systematic increase in FOK from the no- to the fourprime condition, coupled with a progressive decrease in retrieval performance (recall and recognition). Subjects failed to correctly assess the negative impact of semantically related information on subsequent recall, and probably relied on an evaluation of the activation level of the category for FOK assessments.
\end{abstract}

Retrieval blocks frequently occur when related material is presented prior to a target retrieval. This has been demonstrated repeatedly in a number of episodic and semantic memory tasks (Roediger \& Neely, 1982). Are subjects aware of this temporary retrieval interference? Research on the feeling-of-knowing (FOK) experience indicates that subjects can accurately assess the ultimate availability of information that is momentarily inaccessible (Blake, 1973; Gruneberg, Monks, \& Sykes, 1977; Hart, 1965, 1967; Koriat, 1976). However, will the typical positive relationship between subject-assessed and actual retrieval probability be observed in a priming situation in which retrieval ability has been hindered by an experimental manipulation?

Both experiments involved four successive stages: (1) a familiarity rating of non-capital cities, with no, two, or four cities from each state; (2) FOK rating for state capital recall; (3) state capital recall; and (4) state capital recognition. The present design departs from the typical FOK paradigm because subjects assess retrieval probabilities for each target prior to an initial retrieval, rather than rating only those items that are not retrievable on the first try.

\section{EXPERIMENT 1}

\section{Method}

Subjects. Sixty introductory psychology students at Southern Methodist University participated voluntarily, with extra course credit being given as an incentive.

Materials. On the basis of a pilot investigation, seven states were eliminated because the state capital retrieval level was too low (under $4 \%$ ), and one state was eliminated because its capital was too familiar

Experiment 1 was conducted as a master's thesis by the second author. Carrie K. Bradley is now at the University of Texas at Dallas. Requests for reprints should be sent to Alan S. Brown, Department of Psychology, Southern Methodist University, Dallas, TX 75275.
(Texas). The World Book Encyclopedia (1970) was consulted to identify the four largest non-capital cities in each of the 42 remaining states.

Design. There were three prime conditions: each state had no, two, or four non-capital cities shown in the preliminary rating (or priming) task. For the four-city prime states, all four non-capital cities were shown to the subjects. For the two-city prime states, two of the four cities were selected randomly.

The 42 states were rank-ordered by state capital recall frequency, and within each three successive states one was assigned randomly to each of three sets. All 14 states within each set served in the same prime condition (no, two, or four) for a given subject. Three forms of the presentation series were constructed to balance each set of states with each prime condition across subjects.

Procedure. The subjects were tested individually, with 20 subjects given each of the three forms. The subjects were assigned to form on a block-randomized basis as they appeared for the investigation.

For the first task, the subjects were shown a list of 84 non-capital cities: four cities from 14 states, two cities from another 14 states, and no cities from the remaining 14 states. Each city was accompanied by its state name (i.e., Wichita, Kansas). One city from each state in the four-city prime condition was assigned randomly to each quarter of the presentation sequence, and one city from each state in the two-city prime condition was assigned randomly to each half. The subjects indicated how frequently they had heard of each city by circling a number beside it: $1=$ never, $2=$ infrequently, $3=$ occasionally, $4=$ frequently, $5=$ very often, and $6=$ constantly. The subjects were allowed $15 \mathrm{~min}$ for this task.

For the second task, the subjects were given a listing of the 42 states in a random order, and were asked to indicate their FOK for state capital recall by circling a number beside each state: $1=$ don't know, $2=$ vaguely sure, $3=$ somewhat sure, $4=$ moderately sure, $5=$ very sure, and $6=$ positive. The subjects were asked to respond rapidly, on the basis of a first impression, without trying to recall the capital city. They were informed that no capital cities had been shown during the previous task.

After the FOK task, the subjects were given another sheet with the 42 states listed in the same order as in the previous task, and were asked to write the capital city next to each state. They were instructed to write something on each blank, even if it was only a guess.

After the recall sheets had been collected, the subjects were given a forced-choice recognition task. Each state was shown on a separate slide, with five cities listed vertically under it: the four non-capital cities plus the capital city. The capital city appeared in each of the five positions approximately the same number of times across states. Seven states from each of the three sets were assigned randomly to each half of the 
recognition series, and the order of the states within each half was determined randomly.

\section{Results and Discussion}

The following response measures were computed for each subject, for each prime condition: median FOK rating, total recall, and total correct recognition. Medians were computed on the FOK data because the distribution was positively skewed (see Winer, 1971, p. 400). Since the primary analytical interest concerned systematic increases or decreases across the three prime conditions, linear trend tests were applied to each dependent measure.

The means of the medians across prime conditions were 2.37 for no-city, 2.48 for two-city, and 2.60 for four-city primes. Although there was a systematic increase in FOK from no to four primes, the linear trend was nonsignificant $[F(1,118)=2.69, p=.09]$. Looking next at the recall data, the mean correct recall was 3.53 for no-city, 3.33 for two-city, and 3.02 for four-city primes. This systematic decrease from no to four primes resulted in a significant linear trend $[\mathrm{F}(1,118)=3.97, \mathrm{p}=.05]$. Turning to recognition performance, the mean total correct recognition was 7.07 for no-city, 6.40 for two-city, and 5.83 for four-city primes. As with recall, the decrease from the no- to four-city prime conditions was significant in the linear trend test $[\mathrm{F}(1,118)=16.99, \mathrm{p}=.01]$.

The outcome of this first study is important in two respects. First, there was an inverse, rather than the usual direct, relationship between FOK assessments and actual retrieval ability across prime conditions. That is, as the number of city primes increased, there was a (nonsignificant) increase in confidence that was paralleled by a (significant) decrease in recall and recognition ability. This outcome stands in marked contrast to prior investigations showing that subjects can accurately make FOK assessments concerning their memory ability (Blake, 1973; Gruneberg et al., 1977; Hart, 1965, 1967; Koriat, 1976).

Although the outcome of Experiment 1 suggests an inverse relationship between FOK and retrievability, this must remain speculative because of the lack of a significant linear trend for FOK ratings. In light of this, a second study was conducted in an attempt to replicate the initial findings.

\section{EXPERIMENT 2}

\footnotetext{
Method

Subjects. Sixty-five introductory psychology students at Southern Methodist University served in the investigation on a voluntary basis, with extra course credit being given as an incentive.

Materials and Design. Experiment 2 was a replication of Experiment 1 , with respect to materials and design.

Procedure. The procedure was the same as that in Experiment 1, with the following exceptions: (1) The subjects were tested in groups, and (2) the stimuli were presented individually via slide (rather than grouped on sheets) in the prime presentation and FOK rating tasks. The groups of subjects varied in size from 8 to 12 and were assigned to form on a block-randomized basis, such that within each three successive groups, one was assigned randomly to each form. This resulted in 20,22, and 23 subjects in Forms 1, 2, and 3, respectively.
}

\section{Results and Discussion}

In general, the outcome of Experiment 2 duplicated that of Experiment 1: FOK increased, whereas recall and recognition decreased, as the number of primes increased. For the median FOK ratings, the means were 3.52, 3.82, and 3.92 for the no-, two-, and four-city prime conditions, respectively. A significant linear trend test confirmed the reliability of this increase $[\mathrm{F}(1,128)=4.50$, $\mathrm{p}=.04]$. For capital recall, the means were $3.58,3.26$, and 3.23 for the no-, two-, and four-city prime conditions, respectively. This decrease did not result in a significant linear trend $[F(1,128)=2.56, p=.11]$. Finally, the correct recognition scores were 7.72, 7.31, and 7.29 for the no-, two-, and four-city prime conditions, respectively. As with the recall data, the linear trend test was nonsignificant $[\mathrm{F}(1,128)=2.12, \mathrm{p}=.15]$.

Although the significance of the linear trend tests was not consistent from Experiment 1 to Experiment 2, the replication in Experiment 2 of the data trends produced in Experiment 1 strongly supports the empirical reliability of these effects. Guilford $(1965$, p. 248) provided a method for combining independent replications into one statistical test in order to determine whether an effect is significant in a more global sense. This, of course, assumes that the effect is in the same direction in each replication (which it is in the present design). For this analysis, the following formula is used: $\chi^{2}=$ $-4.605 \log \left(\mathrm{p}_{1} \mathrm{p}_{2}\right)$, where $\mathrm{p}$ is the exact probability of the particular test statistic. When the formula is applied to the combined probabilities, the obtained chi-square values $(\mathrm{df}=1)$ are significant for all three tests at the .01 level: $11.51,10.41$, and 17.64 for FOK, recall, and recognition, respectively.

\section{GENERAL DISCUSSION}

The outcome of this set of investigations provides a striking exception to the usually direct correspondence between a person's self-assessed and actual ability to retrieve items from memory (Blake, 1973; Gruneberg et al., 1977; Hart, 1965, 1967; Koriat, 1976). The present study suggests that when subjects receive primes in a certain category, this enhances their confidence in the accessibility of related material, while actually decreasing this ability. It is remarkable that this diminished memory ability was revealed in a recognition test, because subjects knew that those distractor cities shown in the familiarity task (Stage 1) were not capitals. This should have yielded better performance in the twoprime and four-prime conditions (than in the no-prime condition) because subjects could eliminate certain cities on the basis of having been recently exposed to them. However, the opposite outcome occurred.

One clear departure of the present investigation from prior FOK research is the requirement that subjects assess their memory store prior to an initial recall effort. In the past, researchers have examined recognition FOK of nonrecalled items, whereas the present investigation focused on recall FOK of to-be-recalled items. Since the highest-confidence items are usually recalled, and these are excluded from recognition FOK assessments used in previous studies, the present approach of examining recall FOK included a broader range of items and should have shown a more precise correspondence between memory contents and memory evaluation. Therefore, it is ironic that a negative relationship was actually found.

An important question concerning the present results is the mechanism whereby subjects make their recallability assessments. A likely possibility is that it is based on an evaluation of the momentary activation 
level of the category, which is the sum of the exemplars currently in an elevated state of activity. This process may be similar to the availability heuristic (Tversky \& Kahneman, 1973), in which subjects estimate categorical frequency on the basis of how readily instances become available when they try to generate them. Alternatively, the subjects in our experiments may simply have responded to the frequency of exposure to the state name and have based their confidence estimate for state capital recallability on a situationally incremented state frequency attribute (Bacon, 1979; Hasher, Goldstein, \& Toppino, 1977).

The investigation may also shed some light on the tip-of-the-tongue (TOT) experience. In the present paradigm, there was an inversion of the usually direct relationship between the subjective assessment and actual retrievability, which was apparently due to the heightened availability of related information. A possible parallel exists in the TOT experience, in which there is an elevated feeling of confidence accompanied by a temporary impairment of retrievability (R. Brown \& McNeill, 1966; Freedman \& Landauer, 1966). A. S. Brown $(1979,1981)$ and Roediger (1974) have speculated that, in the TOT state, the subject is blocked from retrieving the target item because of activation of related (and inhibiting) material. As in the present design, the subject may be aware of the activation of related memorial information, and be making an erroneously high confidence assessment on this basis.

\section{REFERENCES}

BAcon, F. T. (1979). Credibility of repeated statements: Memory for trivia. Journal of Experimental Psychology: Human Learning and Memory, 5, 241-252.

BLAKE, M. (1973). Prediction of recognition when recall fails: Exploring the feeling-of-knowing phenomenon. Journal of Verbal Learning and Verbal Behavior, 12, 311-319.

Brown, A. S. (1979). Priming effects in semantic memory retrieval processes. Journal of Experimental Psychology: Human Learning and Memory, 5, 65-77.
Brown, A. S. (1981). Inhibition in cued retrieval. Journal of Experimental Psychology: Human Learning and Memory, 7, 204-215.

Brown, R., \& MCNeILL, D. (1965). The "tip of the tongue" phenomenon. Journal of Verbal Learning and Verbal Behavior, 5, 325-337.

Freedman, J. L., \& Landauer, T. K. (1966). Retrieval of long-term memory: "Tip-of-the-tongue", phenomenon. Psychonomic Science, 4, 309-310.

Gruneberg, M. M., Monks, J., \& Sykes, R. N. (1977). Some methodological problems with feeling-of-knowing studies. Acta Psychologica, 41, 365-371.

GUILFORD, J. P. (1965). Fundamental statistics in psychology and education. New York: McGraw-Hill.

HART, J. T. (1965). Memory and the feeling-of-knowing experience. Journal of Educational Psychology, 56, 208-216.

HART, J. T. (1967). Memory and the memory-monitoring process. Journal of Verbal Learning and Verbal Behavior, 6, 685-691.

Hasher, L., Goldstein, D., \& Toppino, T. (1977). Frequency and the conference of referential validity. Journal of Verbal Learning and Verbal Behavior, 16, 107-112.

Koriat, A. (1976). Another look at the relationship between phonetic symbolism and the feeling-of-knowing. Memory \& Cognition, 4, 244-248.

Roediger, H. L. (1974). Inhibiting effects of recall. Memory \& Cognition, 2, 261-269.

Roediger, H. L., \& NeELy, J. H. (1982). Retrieval blocks in episodic and semantic memory. Canadian Journal of Psychology, 36, 213-242.

TVersKy, A., \& KahNeman, D. (1973). Availability: A heuristic for judging frequency and probability. Cognitive Psychology, 5, 207-232.

WINER, B. J. (1971). Statistical principles in experimental design. New York: McGraw-Hill.

THE WORLD BOOK ENCYCLOPEDIA. (1970). New York: World Book, Inc.

(Manuscript received for publication November 13, 1984.) 